\title{
DIVERSIFICAÇÃO E ESPECIALIZAÇÃO PRODUTIVA NA GERAÇÃO DE INOVAÇÃO TECNOLÓGICA: UMA APLICAÇÃO PARA OS ESTADOS BRASILEIROS
}

\author{
Domitila Santos Bahia \\ Doutoranda em economia do Programa de Pós-Graduação em Economia da Universidade Federal de \\ Juiz de Fora - UFJF \\ domitilabahia@gmail.com (Brasil)
}

\section{Armando Vaz Sampaio}

Doutor em Ciências (Economia Aplicada) pela Universidade de São Paulo - USP Professor da Universidade Federal do Paraná - UFPR

avsampaio@ufpr.br (Brasil)

\section{RESUMO}

O principal objetivo dessa pesquisa é investigar o quão sensível é a geração de inovação dos estados brasileiros às influências das externalidades de diversificação e de especialização industrial, no período compreendido entre 2001-2011. Além disso, estudar o comportamento da inovação através do território permite a inferência de como políticas públicas de fomento à ciência e tecnologia tem agido no Brasil. Outros fatores determinantes da inovação são também considerados, como a capacidade de investimento em ciência e tecnologia dos estados, o nível de escolaridade e a defasagem temporal da inovação. Como medida de geração de inovação, são utilizados os depósitos de patentes per capita, a fim de mensurar a capacidade tecnológica de cada estado. A base de dados utilizada neste trabalho consiste na conjugação de dados provenientes do Instituto Brasileiro de Geografia e Estatística (IBGE). dos dados de depósitos de patentes do Instituto Nacional de Propriedade Industrial (INPI), de dados sobre gastos com ciência e tecnologia do Ministério da Ciência, Tecnologia e Inovação (MCTI) e dados sobre capital humano do Ministério da Educação (MEC). A metodologia utilizada aborda a Análise Exploratória de Dados Espaciais (AEDE) e modelos de regressão espacial com dados em painel. Tais procedimentos permitiram acompanhar a trajetória da inovação através do território no período em análise e foram estudados os principais marcos legais da inovação brasileira a fim de se estabelecer se esses esforços contribuíram para o fortalecimento da política regional de inovação.

Palavras-chave: Inovação; Patentes; Estados Brasileiros; Modelos em painel de dados com dependência espacial. 


\section{INTRODUÇÃO}

A motivação desta pesquisa está na necessidade de se aprofundar e compreender a relação entre difusão de conhecimento tecnológico e geração regional de inovações. Esta temática tem importância reconhecida por diversos autores (Feldman e Audretsch, 1999; Griliches, 1990; Cabrer-Borrás e Serrano-Domingo, 2007), principalmente pelo fato de que a inovação e o progresso tecnológico influenciam diretamente o crescimento econômico.

As atividades de inovação tecnológica são o conjuto de etapas científicas, tecnológicas, organizativas, financeiras e comerciais, incluindo os investimentos em novos conhecimentos, que levam ou tentam levar à implementação de produtos e processos novos ou melhorados (OECD, 2002) e são estimuladas pela geração e transferência de conhecimento, que transbordam (efeito spillover) entre as firmas e instituições. A geração de capital humano, presença de instituições de pesquisa e os esforços em pesquisa e desenvolvimento também contribuem no processo de geração de inovação.

Como forma de medir a geração de inovação, pode-se observar a quantidade de patentes depositadas em determinado período. No Brasil, de acordo com o INPI (Instituto Nacional da Propriedade Intelectual), em 2011, foram realizados por residentes 7.764 depósitos de patentes. Desses, 3.296 pedidos vieram do estado de São Paulo. 128 depósitos de patentes foram realizados em 2011 pelos sete estados da Região Norte do Brasil, enquanto que os estados da Região Sul somaram 2.037 depósitos de patentes.

Esses dados corroboram com a hipótese de que a inovação e os transbordamentos de conhecimento não se distribuem de forma equânime entre as regiões, estão reunidos onde a produção é também geograficamente concentrada. Marshall (1982) é um dos precursores na literatura sobre economias de aglomeração e ressalta as vantagens da concentração de indústrias especializadas em certas localidades.

A literatura considera que há dois tipos de spillover: o de especialização, embasado nas teorias de Marshall (1982), Arrow (1962) e Romer (1986), constituindo o acrônimo MAR e o da diversificação, defendido por Jacobs (1969).

Para Jacobs (1969), a interação entre as pessoas gerando novas ideias e produtos explica o processo de difusão da inovação e os transbordamentos proporcionados pelas indústrias de diversos setores.

Revista de Administração e Inovação, São Paulo, v. 12, n.3 p. 109-134, jul./set. 2015. 
Por spillover de diversificação, entende-se a troca complementar de conhecimento através de diversas firmas e agentes econômicos que buscam facilitar as buscas e experimentos na inovação, favorecendo a criação de novas ideias por causa da variedade e diversidade dos setores industriais localizados próximos.

As externalidades de especialização, associadas ao aumento da concentração de uma indústria específica, dentro do espaço geográfico de uma região, facilitam os transbordamentos de conhecimento entre as firmas. As investigações sobre a relação entre inovação e as externalidades espaciais permanecem inconclusivas quanto ao efeito da especialização e da diversificação das estruturas locais produtivas sobre a capacidade de inovação.

O objetivo principal deste trabalho é analisar os determinantes da inovação tecnológica nos estados brasileiros entre os anos de 2001 a 2011 e posteriormente, avaliar em que medida a especialização e a diversificação industrial interferem na geração de inovação pelos estados.

\section{REVISÃO BIBLIOGRÁFICA}

Desde que a inovação e o progresso tecnológico tiveram sua importância reconhecida pela literatura acadêmica como elementos do processo de crescimento econômico, os fatores determinantes desses componentes tem sido amplamente discutidos.

O destaque dado por Schumpeter (1961) à capacidade e à iniciativa dos empresários em consonância com as atividades científicas é justamente porque o empreendedorismo poderia ser o condutor do crescimento econômico através de inovações tecnológicas.

Essas inovações podem ser exemplificadas como a introdução de um novo bem no mercado ou de um aprimoramento de um bem existente, da introdução de um novo método de produção ou nova maneira de comercialização de um produto, abertura de um novo mercado, conquista de uma nova fonte de oferta de matérias-primas e pelo estabelecimento de uma nova organização de qualquer indústria (Schumpeter, 1961).

De acordo com Vargas (2002), a geração de inovação não é um fenômeno isolado no tempo e no espaço, mas sim o resultado de trajetórias cumulativas e construídas historicamente, de acordo com as especificidades institucionais e padrões de especialização econômica inerentes a um determinado contexto espacial ou setorial.

Revista de Administração e Inovação, São Paulo, v. 12, n.3 p. 109-134, jul./set. 2015. 
À luz desses conceitos, é possível fazer duas observações: 1) O conhecimento tecnológico é localizado e sua propagação ocorre lentamente e 2) capacidade de aprendizado é de extrema importância para o desenvolvimento de novas tecnologias.

Qualquer inovação importante baseia-se num estoque de conhecimento, o que denota o conceito de trajetórias pré-estabelecidas e persistência temporal. Mas a capacidade de inovar com sucesso depende de forma crescente do uso de todo esse corpo de conhecimentos estruturados, antigos e novos (Steinmueller, 1994). Ou seja, apenas o conhecimento e a geração de inovação prévios não são suficientes para que a trajetória seja contínua.

Inovação tecnológica é entendida como o processo que determina a capacidade de produzir novos produtos (ou novos processos) e o desenvolvimento tecnológico de uma economia e é um fenômeno complexo e difícil de ser mensurado (Cabrer-Borrás \& Serrano-Domingo, 2007). A medida de inovação tecnológica, através da quantidade de patentes, não é consensual, pois ainda há debates sobre a representação do estado real da inovação e sobre quais aspectos da atividade econômica conseguem ser capturados por esses dados. As ressalvas residem no fato de que nem todas as invenções chegam a ser patenteadas e nem todas as patentes se tornarão inovações incorporadas pela indústria, além de existir diferenças setoriais e diferenças em termo de conteúdo inovador em cada patente depositada.

Evidências empíricas mostram que a produtividade é influenciada pela apropriação de conhecimento, uma vez que um sistema consiso de proteção intelectual encoraja o progresso tecnológico e a invenção, através da concessão do monopólio de exploração destas temporariamente ao inventor.

Fora isso, a relação entre patentes depositadas e produtividade é questionada em países de baixa renda (Penrose, 1973), uma vez que a importação de bens de tecnologia e investimentos diretos externos são estimulados pelo baixo nível de proteção intelectual.

No Brasil, até 2003, cerca de 70\% dos depósitos de patentes foram realizados por pessoas físicas. Segundo Albuquerque (1999), essa proporção revela a falta de continuidade nos depósitos e o pequeno envolvimento das firmas em atividades de inovação e se devem, sobretudo, ao subdesenvolvimento do Sistema Nacional de Inovação (SNI).

A relevância do espaço geográfico como elemento que propicia inovação está vinculada ao tipo de conhecimento, que, de acordo com Dosi (1988), pode ser classificado de acordo com os aspectos universal ou específico, articulado ou tácito e público e privado.

Revista de Administração e Inovação, São Paulo, v. 12, n.3 p. 109-134, jul./set. 2015. 
Os spillovers de conhecimento ocorrem porque o conhecimento criado por uma empresa ou qualquer outra organização não fica contido apenas dentro dessas entidades, mas é também aproveitado por outras empresas e organizações. Nesse sentido, o espaço geográfico é determinante em termos de facilitar a inovação e, consequentemente, aumentar a competitivdade de empresas aglomeradas num mesmo território.

Audretsch e Feldman (1996), Gray e Dunning (2000), Glaeser et al. (1992) são autores que pontuam a importância de uma forte capacitação tecnológica local e/ou regional como um determinante do crescimento das cidades e regiões.

As vantagens proporcionadas pela proximidade geográfica causam os efeitos de spillover e encadeamentos, que surgem nas formas de redução nos custos de fornecimento de insumos, formação de mercado regional de trabalho especializado e facilidade de acesso a informações relevantes às novas tecnologias (Lemos et al, 2005b).

Para Audretsch e Feldman (1996), o conceito de economias de aglomeração (clusters) possui como aspecto principal a proximidade territorial de agentes econômicos, políticos e sociais, e envolve diversos tipos de externalidades, que explicam tanto a aglomeração espacial da produção quanto da inovação.

Há três meios para a condução desses spillovers: primeiro, através do setor científico e seu estoque de conhecimento geral e tecnológico; segundo, através do conjunto de conhecimentos específicos da empresa; e terceiro, através das relações entre empresas e universidades (Fischer, 2001).

Para Audretsch (1998), a inovação é mais concentrada nas indústrias em que os transbordamentos de conhecimento são prevalecentes. Habilidades e alto nível de capital humano também aumentam o efeito transbordamento. Nesse sentido, o autor destaca que os fluxos de conhecimento concentrados nas grandes cidades são justificados pelo grande acúmulo de profissionais qualificados, fornecedores e ampla rede de instituições de pesquisa regionais, como universidades e agências de fomento à pesquisa.

\subsection{Principais características da inovação no Brasil}

Como tema central para o desenvolvimento produtivo do país, a transformação da indústria via inovação tem sido requerida mais sistematicamente nos últimos anos para promover maior desenvolvimento econômico e aumento na geração de renda. O Estado vem criando políticas de incentivo à inovação, com o objetivo de engajar as empresas numa mudança de posicionamento na capacidade de desenvolver e implementar novas tecnologias.

Revista de Administração e Inovação, São Paulo, v. 12, n.3 p. 109-134, jul./set. 2015. 
Tais políticas de desenvolvimento industrial, baseadas em inovação e diferenciação de produto, tem se mostrado positivas para o crescimento das firmas brasileiras (Salerno \& Kubota, 2008). Entretanto, o envolvimento de pequenas firmas nas atividades de inovação e a falta de continuidade nos processos de patenteamento das inovações revelam o baixo grau das atividades de $\mathrm{P} \& \mathrm{D}$ realizados pela indústria no geral (De Negri et al, 2005).

Segundo Gonçalves (2007a), o principal determinante do esforço inovador no Brasil é a firma, principalmente as grandes empresas. As inovações no Brasil estão condicionadas ao território, enfatizando a grande dificuldade dos ambientes periféricos em gerar inovação por causa da fragilidade dos seus atores e da pobreza dos seus atributos regionais, redundando em poucas externalidades favoráveis à inovação.

Característica da formação do setor industrial brasileiro, o esgotamento do modelo de substituição de importações dificultou a possibilidade de desenvolver a capacidade tecnológica nacional pelas transformações institucionais ocorridas nas décadas de 80 e 90 . Desse modo, a grande participação de empresas estrangeiras nesse processo abreviou os passos da industrialização e facilitou a transferência de tecnologia. Mas para as empresas de capital nacional, esse acesso à tecnologia deuse somente através da importação de máquinas e equipamentos (Pacheco, 2007).

Para Gonçalves (2007a), as firmas industriais brasileiras, em termos de sua capacidade de introduzir novos produtos para o mercado doméstico, dão importância à aquisição externa de tecnologia através de compra de $\mathrm{P} \& \mathrm{D}$, ao licenciamento, à compra de know-how, patentes, marcas registradas, serviços de consultoria e aos acordos de transferência de tecnologia.

Quanto a questão de políticas públicas de inovação, o que se tem no Brasil é algo recente, resultante de legislações da década de 1990 que alteraram a visão de que ciência e tecnologia, no país, era atividade quase exclusiva de institutos e universidades públicas.

O estímulo às atividades de pesquisa e desenvolvimento é legislado principalmente sob a forma de incentivos fiscais de naturezas diversas. A primeira lei sobre o tema, em 1993, dispôs sobre concessões fiscais para a capacitação tecnológica da indústria e da agropecuária e não tinha o propósito específico de gerar inovação.

As proposições de leis anteriores a 1999 foram importantes para a regulação das atividades de ciência e tecnologia, entretanto, quase não afetaram a estrutura de incentivos à inovação, fomento e financiamento às atividades inovadoras.

Revista de Administração e Inovação, São Paulo, v. 12, n.3 p. 109-134, jul./set. 2015. 
O marco legal brasileiro para inovação está alicerçado, resumidamente, sobre a Lei de Inovação Federal (Lei Federal n. ${ }^{\circ} 10.973$ de 02.12.2004), Leis estaduais de inovação e a Lei do Bem (Lei Federal n. ${ }^{\circ} 11.196$ de 21.11.2005).

A Lei de Inovação Federal dispõe sobre incentivos à inovação e à pesquisa científica e tecnológica no ambiente produtivo, estabelecendo medidas de fomento à capacitação com a finalidade de obter autonomia tecnológica e desenvolvimento industrial do país.

A intenção de fomentar a inovação de maneira justa no espaço geográfico é demonstrada ainda na Lei da Inovação, que estabelece que sejam priorizadas, nas regiões menos desenvolvidas do país e na Amazônia, ações que visem a dotar a pesquisa e o sistema produtivo regional de maiores recursos humanos e capacitação tecnológica, assim como assegurar tratamento favorecido a empresas de pequeno porte e dar prioridade às empresas que invistam em pesquisa e desenvolvimento no país, nos casos de aquisição de bens e serviços pelo Poder Público.

As características de assimetria do sistema de inovação brasileiro são conhecidas e tem sido descritas através do confronto entre bons indicadores acadêmicos e índices relativamente piores quanto às atividades de $\mathrm{P} \& \mathrm{D}$ do setor privado.

Para Pacheco (2003), a consolidação da pós-graduação corresponde a um grande esforço de qualificação de pessoal e fortalecimento da pesquisa acadêmica que deveria ter sido acompanhada pelo fortalecimento tecnológico das empresas. Essa dimensão foi sempre a parte frágil do modelo e sua debilidade.

Em 2001, o gasto total do Brasil com P\&D era de 1,04\% do PIB. Segundo Pacheco (2003), um esforço compatível com as dimensões da economia brasileira, mas muito concentrado no setor público.

Para que haja o aumento do gasto privado, é necessário uma ação indutora do setor público, quer na forma de incentivos fiscais, quer na forma de encomendas ou apoio direto à inovação. E também o reforço às externalidades do conhecimento e forte ênfase na cooperação entre empresas e instituições do sistema de inovação (Pacheco, 2003). Isto porque é reconhecido que os mecanismos de mercado são ineficientes para viabilizar um patamar adequado de gasto, exigindo um papel ativo dos governos, na forma de parcerias público-privado, fomento direto e incentivos fiscais.

\section{ANÁLISE EXPLORATÓRIA DOS DADOS ESPACIAIS}

A análise exploratória (AEDE) é um conjunto de técnicas que descrevem e ilustram distribuições espaciais, identificando assim localidades atípicas (outliers espaciais), descobrindo 
padrões de associação espacial e sugerindo regimes espaciais e outras formas de instabilidade espacial. O paradigma dessa análise é baseado na intenção de deixar os dados falam por si e com finalidade de impor o mínimo de estrutura prévia sobre eles quanto possível. (Anselin, 1999).

Para Anselin e Bera (1998), autocorrelação espacial significa a ocorrência da observação de valores similares em localidades próximas ou a ocorrência de valores dissimilares em torno de um valor de referência. Pode-se construir um coeficiente de autocorrelação espacial tendo-se necessariamente três elementos: uma medida de covariância, uma medida de variância dos dados e uma matriz de ponderação espacial $(W)$. As estatísticas de autocorrelação espacial mais adotadas na literatura são o $I$ de Moran, $c$ de Geary e $G$ de Getis-Ord.

A matriz de pesos espaciais (ou matriz $W$ ) tem por principal finalidade a viabilização da abordagem paramétrica das relações de dependência espacial. Através dessa escolha, é imposto um arranjo para a ocorrência das interações espaciais entre as regiões na forma matricial. Cada conexão entre duas regiões é representada numa célula da matriz $W$, sendo denominada de peso espacial. O importante é estabelecer uma medida factível capaz de mensurar corretamente o grau de conexão entre as regiões. Tais medidas podem ser de caráter geográfico, sócioeconômico ou, ainda, qualquer outro critério relevante para o fenômeno a ser analisado.

Entretanto, a escolha da matriz de pesos espaciais é bastante controversa por não haver um critério consiso para a justificativa da mesma. Muitas vezes a escolha da matriz $W$ é arbitrária e também existe o problema da sensibilidade dos resultados à escolha da matriz.

De acordo com Almeida (2012), a matriz de ponderação espacial deveria ser construída com o intuito de capturar toda a autocorrelação espacial subjacente ao fenômeno em estudo.

Para LeSage e Pace (2014), tal hipótese seria um mito que perpetua a ideia da necessidade de ajustamento das especificações da matriz $\mathrm{W}$, porque as estimativas e inferências podem ou não ser sensíveis a pequenas mudanças nessas especificações. Para os autores, a alteração da matriz de pesos espaciais pode causar mudanças nas medidas de dispersão (estatística $t$ ), mas não causaria diferenças significativas nos coeficientes $\beta$. De modo geral, conclui-se que a existência de sensibilidade a mudanças na matriz $W$ pode indicar erro na especificação do modelo. Se o mito fosse comprovado, então modelos de regressão espacial poderiam ser considerados mal-condicionados e representariam um método fora de propósito para a análise de relações envolvendo dados espacialmente dependentes (Lesage \& Pace, 2014).

Para a efetiva escolha da matriz de ponderação espacial, tentando amenizar a escolha arbitrária, utilizou-se o critério descrito por Baumont (2004), sendo criadas matrizes espaciais do tipo queen, do 
tipo torre e de $\mathrm{k}$ vizinhos mais próximos $(\mathrm{k}=1, \mathrm{k}=2, \mathrm{k}=3, \mathrm{k}=4, \mathrm{k}=5, \mathrm{k}=10, \mathrm{k}=15$ e k=20) e escolhida aquela com maior $I$ de Moran significativo.

No caso desta pesquisa, a matriz escolhida foi a matriz de distância geográfica dos $\mathrm{k}=2$ vizinhos mais próximos. A ideia básica é a de que regiões mais próximas geográficamente tem maior interação espacial. Segundo Almeida (2012), a vantagem deste tipo de convenção é combater o desequilíbrio da conectividade de uma matriz, pois todas as unidades espaciais terão o mesmo número de vizinhos. Além disso, ao adotar esse tipo de matriz, é garantido que todas as regiões terão vizinhos, ou seja, não haverá "ilhas".

A autocorrelação espacial, positiva ou negativa, está relacionada à similaridade ou dissimilaridade dos valores das variáveis entre as regiões e pode ser verificada pelo coeficiente $I$ de Moran que fornece, em resumo, três informações importantes. O nível de significância da estatística mostra se os dados estão ou não distribuídos aleatoriamente. O sinal do coeficiente, desde que significativo, indica a concentração (sinal positivo) e a dispersão (sinal negativo) através das regiões. A magnitude da estatística fornece a força da autocorrelação espacial. Quanto mais próximo de 1 for o valor do coeficiente I, mais concentrados estão os dados. Quanto mais próximos de -1, estão mais dispersos no espaço.

A ideia da mensuração da correlação espacial entre a variável de interesse e de sua defasagem espacial pode ser estendida para o caso de duas ou mais variáveis. Intuitivamente, ao calcular a autocorrelação espacial multivariada, o que se pretende é verificar se os valores de determinada variável em certa região são afetados por outras variáveis observadas em regiões vizinhas.

Por definição, outliers são as observações que não seguem o mesmo padrão que a maioria dos dados da amostra. Podem ser distinguidos entre outliers globais e outliers locais. Observações muito dissimilares (valores mais baixos ou mais altos) dentro de uma amostra são chamadas outliers globais e as observações que não seguem o mesmo padrão de dependência espacial do que a maioria dos dados da amostra são os outliers locais ou outliers espaciais. A ocorrência desse tipo de dados pode exercer uma influência espúria sobre a medida global de autocorrelação espacial, podendo deturpar a estimativa do teste (Almeida, 2012). Além disso, pode significar, segundo Anselin (1996), má especificação da matriz $W$ ou de inadequada escala espacial dos dados.

Revista de Administração e Inovação, São Paulo, v. 12, n.3 p. 109-134, jul./set. 2015. 


\section{MODELO DE REGRESSÃO COM DADOS EM PAINEL}

O método adotado para este trabalho foi o modelo de dados em painel, de forma dinâmica no tempo e no espaço. A justificativa para a escolha pelo modelo de dados em painel é decorrente da utilidade em lidar com o problema de variáveis omitidas, além do que esse tipo de modelo é capaz de acomodar a heterogeneidade espacial que é representada por regiões específicas, não observáveis e com interceptos de tempo que não variam.

Particularmente, os modelos de dados em painel com defasagem espacial podem apresentar problemas como a heterogeneidade espacial e a dependência espacial.

A heterogeneidade espacial surge quando o território em análise possui unidades espaciais muito diferentes entre si e pode resumir a falta de estabilidade do comportamento econômico através do espaço, quando é possível notar a existência de padrões de agrupamento nos dados (Almeida, 2012).

Já a dependência espacial pode estar alojada nos resíduos, contaminando as estimativas se não tratada adequadamente. A literatura considera que se não levada em conta, a dependência espacial pode tornar os estimadores da regressão inconsistentes e/ou ineficientes (Anselin, 1988).

Para decidir se existe a necessidade de introduzir alguma defasagem espacial, é preciso checar se os resíduos do modelo convencional de efeitos fixos mostram-se autocorrelacionados espacialmente. Para averiguar a presença de autocorrelação espacial nos resíduos, aplicam-se testes de autocorrelação espacial, tais como o teste $I$ de Moran para os resíduos das unidades de corte transversal para cada período ou testes de multiplicador de Lagrange da defasagem espacial e do erro espacial, propostos por Elhorst (2003). Constatada a presença de erros autocorrelacionados, há a necessidade de tratar essa dependência, incorporando a defasagem espacial ao modelo.

\subsection{Modelo empírico}

Como exposto no início da seção sobre a metodologia desta pesquisa, o referencial empírico será o modelo econométrico espacial para dados em painel proposto por Cabrer-Borrás e SerranoDomingo (2007).

$$
\begin{aligned}
& I_{t}=\alpha+\rho W_{1} I_{t}+\beta_{1} I_{t-1}+\beta_{2} P D_{t-1}+Z_{t} \delta+W_{1} F S_{t} \tau+\varepsilon_{t} \\
& \varepsilon_{t}=\lambda W_{2} \varepsilon_{t}+u_{t}
\end{aligned}
$$


O termo da equação 1.a $I_{t}=\left(I_{1 t}, \ldots, I_{N t}\right)^{\prime}$ é o vetor das patentes per capita, utilizado como proxy da geração de inovação pelos estados $i$ no período $t ; \alpha=\left(\alpha_{1}, \ldots, \alpha_{N}\right)^{\prime}$ é o vetor de efeitos fixos específicos para cada estado, $W_{1}$ é a matriz de pesos espaciais utilizada, no qual $W_{1} I_{t}=\left(W_{1} I_{1 t}, \ldots, W_{1} I_{N t}\right)^{\prime}$ é o vetor de defasagem espacial da variável dependente e $\rho$ é o coeficiente de defasagem espacial, captando os efeitos de spillover da geração de inovação; o vetor $I_{t-1}=\left(I_{1 t-1}, \ldots, I_{N t-1}\right)^{\prime}$ é a defasagem temporal da variável inovação e $\beta_{1}$ é o parâmetro a ser estimado. $P D_{t-1}=\left(P D_{1, t-1}, \ldots, P D_{N, t-1}\right)^{\prime}$ é o vetor dos gastos com ciência e tecnologia (inovação e áreas correlatas) realizados pelos estados e $\beta_{2}$ é seu coeficiente a ser estimado; $Z_{t}=\left(Z_{1 t}, \ldots, Z_{N t}\right)^{\prime}$ é a matriz que representa as outras variáveis explicativas $\left(E_{t}, S_{t}, D_{t}\right)$ e $\delta=\left(\delta_{1}, \ldots, \delta_{k}\right)^{\prime}$ são os coeficientes a serem estimados; $W_{1} F S_{t}=\left(W_{1} F S_{1 t}{ }^{\prime}, \ldots, W_{1} F S_{N t}\right)^{\prime}$ expressa a matriz de defasagem espacial das variáveis explicativas, e $\tau=\left(\tau_{1}, \ldots, \tau_{k}\right)^{\prime}$ é o vetor das externalidades dessas variáveis; $\varepsilon_{t}=\left(\varepsilon_{1 t}, \ldots, \varepsilon_{N t}\right)^{\prime}$ é o vetor dos termos de erro autocorrelacionados e $u_{t}=\left(u_{1 t}, \ldots, u_{N t}\right)^{\alpha}$ é o vetor dos termos de erro independentes e identicamente distribuídos (i.i.d.) com variância $\sigma^{2}, \lambda$ é o coeficiente de autocorrelação espacial do termo de erro; $W_{2}$ representa a matriz de pesos espaciais, na qual $W_{2} \varepsilon_{t}=\left(W_{2} \varepsilon_{1 t}, \ldots, W_{2} \varepsilon_{N t}\right)^{\prime}$ é o vetor de defasagem do termo de erro.

\subsection{Base de dados}

A base de dados utilizada neste trabalho é a conjugação de diferentes fontes e organizados para cada estado da federação, totalizando 11 observações na dimensão temporal e 27 observação na cross section (unidades da federação), resultando assim em 297 observações no painel.

O recorte territorial de estados da federação utilizado como unidade de análise foi adotado principalmente pela disponibilidade de dados, que são encontrados agregados por estado da federação.

O uso de cada variável é justificado pelo uso em trabalhos empíricos da mesma temática e de acordo com a teoria adjacente ao modelo. Com exceção da variável $C T_{t-1}$, que representa o gasto empreendido pelos estados em atividades de ciência e tecnologia (P\&D e atividades correlacionadas) e não foi utilizada da mesma maneira que em trabalhos correlatos (Bilbao-Osorio \& Rodríguez-Pose, 2004; Co, 2002; Koo, 2005), já que a variável contempla somente os gastos públicos de investimento em pesquisa e desenvolvimento e exclui os gastos privados realizados dentro das firmas para a geração de inovação. 
A variável dependente é $I_{t}$, uma proxy da capacidade de geração da inovação ou da atividade tecnológica dos estados, e é resultado da divisão do número de depósitos de patentes dos estados, segundo o INPI, pelo total da população dos mesmos, segundo as estimativas de população de cada ano da pesquisa.

A variável dependente defasada no tempo, $I_{t-1}$, representa o total de depósito de patentes dos estados dividido pelo total de sua população, ambos defasados em um período de um ano. Esta variável foi incluída a fim de permitir confirmar a hipótese de que a produção tecnológica regional é dependente de uma trajetória preestabelecida (path-dependece), segundo Arthur (1989).

A variável $C T_{t-1}$ é a variável que revela o valor gasto pelos estados brasileiros com ciência e tecnologia. Essa variável está disponível no site do Ministério da Ciência e Tecnologia e Inovação (MCTI, 2013) e engloba os gastos feitos com P\&D e em áreas científicas e técnicas correlatas (ACTC) feitos pelas Secretarias Estaduais de Ciência e Tecnologia e Institutos de Pesquisa subordinados a elas, pelas Fundações de Amparo à Pesquisa e/ou fundações semelhantes, pelas Empresas Estaduais de Pesquisa Agropecuária e pelos demais institutos de pesquisa das áreas. De acordo com o MCTI (2013), como gastos com pesquisa e desenvolvimento é utilizada a proxy de gastos com pós-graduação dos Institutos de Ensino Superior (IES) e compreendem todo o trabalho criativo efetuado sistematicamente para ampliar a base de conhecimentos científicos e tecnológicos. Já as ACTC são aquelas que apoiam diretamente as atividades de P\&D. O objetivo principal da inclusão desta variável é verificar se a capacidade de realizar $P \& D$ em um período influencia a geração de inovação em um período subsequente.

A variável índice de especialização industrial $S_{t}$ foi construída da seguinte maneira:

$$
S_{t}=\left(\frac{1}{2}\right) \sum_{i}\left|\left(V T I_{i j} / V T I_{i}\right)-\left(V T I_{N j} / V T I_{N}\right)\right|
$$

Onde $i$ representa o estado, $j$ o VTI do setor industrial e $N$ o valor total do VTI do setor industrial. A variável possui uma variação de zero a um, sendo interpretada de maneira que quanto mais próximo de um, maior o grau de especialização em atividades ligadas a um determinado setor industrial. De modo análogo, quanto mais próxima de zero, menos especializada será a composição industrial do estado selecionado.

Os efeitos da especialização industrial são vinculados à acumulação de capital humano e aos spillovers de inovação tecnológica entre as firmas de um mesmo setor da indústria, atingindo assim,

Revista de Administração e Inovação, São Paulo, v. 12, n.3 p. 109-134, jul./set. 2015. 
retornos crescentes de escala ao final da produção e uma melhor produtividade (Romer, 1990). O coeficiente positivo dessa variável indica que existe especialização industrial nos estados.

A variável índice de diversificação também é construída e representa o grau de diversificação dos setores industriais. Sua fórmula é a que se segue:

$$
D_{t}=\Sigma_{j}\left(V T I_{i j} / V T I_{i}\right)^{2}
$$

Os valores assumidos por $D_{t}$ variam de zero a um, e podem ser interpretados de forma tal que quanto mais próximo de zero, maior é o grau de diversidade das indústrias do estado.

A última variável explicativa do modelo é o nível de escolaridade, $E_{t}$, e será utilizada como proxy desta a razão entre o número de pessoas matriculadas em instituições de ensino superior e a população total do estado. A fonte desses dados é o Ministério da Educação. A intenção é avaliar o papel da dotação de capital humano sobre as atividades de inovação estadual.

\section{RESULTADOS}

A formalização dos indícios de autocorrelação espacial está nos cálculos das estatísticas do $I$ de Moran, que possibilitam analisar a existência e a força da autocorrelação espacial entre os dados da amostra da variável patente per capita.

A tabela 1 apresenta o índice $I$ de Moran das patentes per capita para os anos de 2001 a 2011, revelando a existência da autocorrelação espacial positiva ou negativa para todos os anos. Isso significa que os valores de patentes per capita seguem um padrão de dependência espacial.

De acordo com a tabela 1, pode-se perceber que todos os coeficientes são estatisticamente significativos ao nível de $5 \%$ de significância.

\section{Tabela 1}

Índice de Moran das patentes per capita no período 2001-2011

\begin{tabular}{|c|c|c|c|c|c|}
\hline Ano & $I$ de Moran & $\mathrm{E}(I)$ & Erro padrão $I$ & t-stat $I$ & $\mathrm{p}$-value $I$ \\
\hline 2001 & 0,814 & $-0,0385$ & 0,124 & 6,57 & $7,05 \cdot 10^{-7}$ \\
\hline 2002 & 0,785 & $-0,0385$ & 0,129 & 6,1 & $2,24 \cdot 10^{-6}$ \\
\hline 2003 & 0,869 & $-0,0385$ & 0,119 & 7,32 & $1,15 \cdot 10^{-7}$ \\
\hline 2004 & 0,857 & $-0,0385$ & 0,117 & 7,33 & $1,1 \cdot 10^{-7}$ \\
\hline 2005 & 0,84 & $-0,0385$ & 0,123 & 6,81 & $3,9 \cdot 10^{-7}$ \\
\hline 2006 & 0,792 & $-0,0385$ & 0,125 & 6,34 & $1,22 \cdot 10^{-6}$ \\
\hline
\end{tabular}


Diversificação e especialização produtiva na geração de inovação tecnológica: uma aplicação para os estados

brasileiros

\begin{tabular}{|l|c|c|c|c|c|}
\hline 2007 & 0,887 & $-0,0385$ & 0,108 & 8,23 & $1,39 \cdot 10^{-8}$ \\
\hline 2008 & 0,879 & $-0,0385$ & 0,114 & 7,69 & $4,77 \cdot 10^{-8}$ \\
\hline 2009 & 0,832 & $-0,0385$ & 0,125 & 6,64 & $5,86 \cdot 10^{-7}$ \\
\hline 2010 & 0,843 & $-0,0385$ & 0,119 & 7,1 & $1,91 \cdot 10^{-7}$ \\
\hline 2011 & 0,82 & $-0,0385$ & 0,118 & 6,96 & $2,72 \cdot 10^{-7}$ \\
\hline
\end{tabular}

Observação: a matriz de ponderação espacial utilizada foi do tipo $\mathrm{K}=2$ vizinhos. Fonte: Elaboração própria com base no software GeoDa 1.4.6

Ao testar a hipótese de autocorrelação espacial da variável, o valor esperado do coeficiente, $E(I)=-0,0385$, está situado abaixo dos valores calculados, o que significa autocorrelação espacial positiva. Em outras palavras, há similaridade entre os estados: estados com alto valor de patentes per capita tem ao menos dois estados vizinhos com alto valor de patentes per capita, e estados com baixo valor de patentes per capita são vizinhos de estados com baixo valor de patentes per capita.

Através da figura 2, é possível analisar a concentração da inovação, medida pelas patentes per capita, ao longo dos onze anos pesquisados. Para a montagem desse mapa, foi utilizada a média simples das patentes per capita dos estados no período, e, claramente, o que se percebeu foi a concentração da geração de inovação nos estados do sul e sudeste do Brasil.

\section{Figura 2}

Mapa concentração média da geração de inovação no período de 2001 a 2011

[1,8 e-006: 2,6075 e-005] (19)

[2,6075 e -005: 5,035 e-005] (3)

[5,035 e-005: 7,4625 e-005] (3)

[7,4625 e-005: 9,89 e-005] (2)

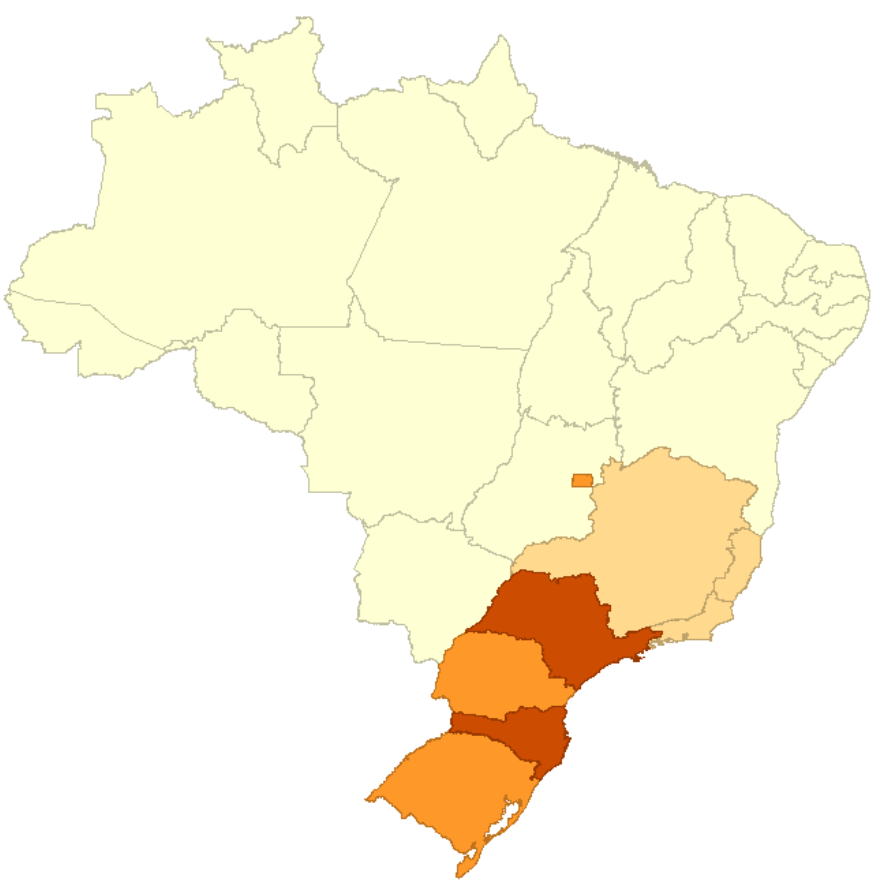

Fonte: elaboração própria com base no software GeoDa 1.4.6 
Através da análise dos indicadores locais de associação espacial, pode-se concluir que o padrão de associação espacial não se alterou significativamente durante os onze anos da análise. Os estados de São Paulo, Paraná, Santa Catarina e Rio Grande do Sul formaram um cluster do tipo alto-alto em todos os anos, ou seja, no período em questão, esses estados tiveram alto valor de geração de inovação e estiveram próximos geograficamente a estados também com alto valor de geração de inovação.

As mudanças espaciais da distribuição da inovação no Brasil ocorreram somente na situação de cluster do tipo baixo-baixo, no qual um estado com baixo valor de patentes per capita conta com dois estados vizinhos com baixos valores de patentes per capita também. Os estados que se revezaram nessa situação ao longo dos anos foram Amazonas, Amapá, Pará e Bahia.

Já a análise da autocorrelação espacial bivariada, verificou a existência de padrões de associação espacial entre duas variáveis, isto é, se a variável patente per capita guarda alguma relação com os valores das outras variáveis de outros estados.

De acordo com os resultados obtidos, é possível afirmar que existe uma associação espacial negativa significativa para todo o período entre as variáveis patentes per capita e o índice de diversificação.

Isso quer dizer que estados com alto valor de geração de inovação estão rodeados de estados com baixos índices de diversificação, cuja construção já detalhada metodologicamente mostra que baixos valores do índice revela alto grau de diversificação industrial. De maneira análoga, estados com baixos valores de patentes per capita são vizinhos de estados com alto índice de diversificação industrial.

Deste modo, a atividade inovativa está positivamente relacionada com a localidades que apresentem alto grau de diversificação industrial, o que corrobora a hipótese de Jacobs (1969).

Em todos os anos do período, os estados de São Paulo, Paraná, Santa Catarina e Rio Grande do Sul formaram clusters do tipo alto-baixo, revelando que estes estados possuem alto desempenho na geração de inovação e estão rodeados por estados com baixo índice de diversificação industrial. O estado do Mato Grosso do Sul formou um cluster do tipo baixo-baixo em todos os anos do período, exatamente por que possui baixa geração de inovação e possui ao menos dois estados vizinhos com indústrias bastante diversificadas.

O mesmo exercício foi realizado confrontando as variáveis patentes per capita e o índice de especialização com a finalidade de testar a hipótese MAR, de que uma indústria altamente especializada favoreceria o surgimento de inovação.

Os resultados mostram coeficientes estatisticamente significativos, revelando que existe associação espacial entre geração de inovação de um estado e o grau de especialização industrial de

Revista de Administração e Inovação, São Paulo, v. 12, n.3 p. 109-134, jul./set. 2015. 
sua vizinhança. Essa associação é, no geral, negativa, ou seja, estados com alto valor de patentes per capita tem ao menos dois vizinhos com baixo índice de especialização industrial. Isso quer dizer que indústrias mais especializadas não contribuem para o aumento ou melhoramento do conjunto atuante na geração de inovação e descarta, a priori, para os estados brasileiros no período de 2001 a 2011, a hipótese do tipo MAR.

A interpretação para essa formação pode estar associada ao fato de que a construção do índice de especialização, ao tomar valores agregados, que é o caso desta pesquisa, não revela que alta especialização da indústria nesses estados é fruto de poucas oportunidades de crescimento industrial, e muitas vezes refletem tão somente a exploração de recursos naturais e transformação industrial de baixo valor agregado. Definitivamente, para a geração de inovação tecnológica, tal situação não contribui.

\subsection{Resultados econométricos}

Como forma de avaliar a significância das variáveis e ter uma base comparativa para as próximas estimações, primeiramente estimou-se o modelo sem nenhum tipo de efeito (fixo ou aleatório) e sem o efeito da dependência espacial (Pooled OLS).

Neste modelo, as 297 observações do painel serão estimadas juntas, o maior problema deste tipo de estimação para dados em painel é negar a heterogeneidade ou invidualidade que pode existir entre os 27 estados.

\section{Tabela 4}

Resultados dos modelos estimados por MQO e do modelo de efeitos fixos (LSDV)

\begin{tabular}{|c|c|c|}
\hline Variáveis & Modelo (MQO) & Modelo (LSDV) \\
\hline \multirow{2}{*}{ Constante } & $-3,28 \mathrm{E}-06$ & 0,0000191 \\
& $(0,369)$ & $(0,000)$ \\
\hline \multirow{2}{*}{$I_{\mathrm{t}-1}$} & 0,5690773 & 0,1890778 \\
& $(0,000)$ & $-5,79 \mathrm{E}-09$ \\
\hline \multirow{2}{*}{$C T_{t-1}$} & $6,92 \mathrm{E}-10$ & $(0,000)$ \\
\hline \multirow{2}{*}{$S_{t}$} & $(0,753)$ & $-0,0000118$ \\
& 0,0000371 & $(0,054)$ \\
\hline \multirow{2}{*}{$D_{t}$} & $(0,000)$ & $6,53 \mathrm{E}-07$ \\
& $-0,0000441$ & $(0,894)$ \\
\hline \multirow{2}{*}{$E_{t}$} & $(0,000)$ & $3,19 \mathrm{E}-11$ \\
& $4,00 \mathrm{E}-11$ & $(0,000)$ \\
\hline$R^{2}$ & $(0,000)$ & 0,7169 \\
\hline AIC & 0,8021 & $-6981,262$ \\
\hline Teste Jarque Bera & $-6430,1413$ & $64,24 * * *$ \\
\hline
\end{tabular}


\begin{tabular}{l|c|c|}
\hline Teste Hausman & - & 32,36 \\
\hline Nota. Variável dependente: $I_{\text {t. }} * * *$ Resultados estatisticamente significativos ao nível de 5\%. Observação: A \\
probabilidade de $p>t$ encontra-se entre parêntesis. Fonte: Elaboração própria com base no programa Stata 12.
\end{tabular}

$\mathrm{Na}$ tabela 4, encontram-se os resultados das estimações do modelo de dados agrupados por MQO e do modelo de efeitos fixos, sem o tratamento da dependência espacial, estimado por Mínimos Quadrados Variáveis Dummies (LSDV).

É possível observar que, no modelo estimado por MQO, todas as variáveis explicativas apresentaram os sinais esperados e apenas a variável gastos com C\&T não é significativa. O poder explicativo do modelo, medido pelo coeficiente $R^{2}$, é de $80,21 \%$ e a hipótese de não normalidade dos resíduos é confirmada pelo teste Jarque Bera.

Como o modelo teórico incita a existência de efeitos não observados, como a atratividade econômica regional e os incentivos legais e políticos à determinadas atividades econômicas, além da própria hipótese de que o espaço geográfico interfere nas variáveis sob estudo. Assim, a estimação por MQO, na presença de heterogeneidade não observada, não é a melhor opção justamente por produzir estimativas enviesadas.

Isto posto, o teste de Hausman foi realizado para sinalizar qual tratamento seria o mais adequado aos efeitos não observados: efeitos fixos ou efeitos aleatórios. O teste indicou que a estimação por efeitos fixos é a mais adequada.

Os resultados da tabela 4 mostram que, estimado por LSDV, nem todas as variáveis do modelo seguiram os sinais esperados, tampouco foram todas significativas. Nesse caso, a variável que mede os gastos com C\&T apresentou sinal negativo, apesar de ser estatisticamente significativa, o que contraria os pressupostos teóricos deste trabalho. A variável diversificação industrial, não significativa, apresentou sinal inverso do esperado também, assim como a variável especialização.

Diante do exposto, a presença da heterocedasticidade e a não-normalidade dos resíduos pedem, como solução, a estimação pelo método dos Mínimos Quadrados Generalizados Exequíveis (MQGE). 
Feita a estimação cross-section dos termos de erro, a dependência espacial foi testada através do I de Moran para os resíduos da regressão a fim de que fossem capturados os efeitos espaciais não somente nas variáveis explicativas, como também na forma de autocorrelação espacial dos resíduos. Os resultados indicaram a existência de dependência espacial nos anos de 2009 e de 2011, ao nível de 5\% de significância e no ano de 2010, ao nível de $10 \%$ de significância. Isso mostra que existe correlação espacial entre as unidades da cross-section ${ }^{l}$.

Os critérios de seleção dos modelos estimados foram a inexistência de autocorrelação espacial dos resíduos e, em segundo lugar, o menor valor do critério de informação Akaike (AIC) apresentado.

De acordo com os resultados das estimações feitas, o modelo escolhido foi o modelo de Durbin espacial, que inclui o efeito transbordamento espacial tanto pela variável dependente quanto pelas variáveis explicativas e é especificado como:

$$
I_{t}=\alpha+\rho W_{1} I_{t}+\beta_{1} I_{t-1}+\beta_{2} P D_{t-1}+Z_{t} \delta+W_{1} F S_{t} \tau+\varepsilon_{t}
$$

Esse modelo mostra o efeito de vizinhança sobre a geração de inovação entre as regiões, no caso, os estados brasileiros.

Almeida (2012) recomenda que não exista dependência espacial nos resíduos dos modelos. Entretanto, para Cardoso et al. (2013) a literatura ainda está avançando na parte de testes residuais para painéis espaciais. Sen e Bera (2011), por exemplo, mostram que o teste Breusch-Pagan tem "excessivo tamanho" causando "excesso de rejeição" para painéis espaciais, principalmente na presença de crosssection dependence $(C D)$. O teste pode apresentar com muita frequência erro do tipo I, rejeitando a hipótese nula quando ela não deveria ser rejeitada. Provavelmente por conta da explicação de Sen e Bera (2011), todos os modelos rejeitaram a hipótese nula do teste. Por isso, foi escolhido o modelo que mais se aproximou de não rejeitar a hipótese nula, o modelo de Durbin espacial (modelo 5), ainda que este não tenha apresentado o menor valor do critério de Akaike.

\section{ANÁLISE DOS RESULTADOS}

A variável que representa a inércia temporal da geração de inovação $\left(I_{t-1}\right)$ apresentou sinal

\footnotetext{
${ }^{1}$ Cross-section dependence (CD): falta de independência entre as unidades da cross-section.
} 
positivo e significância estatística. Esse resultado vai de encontro com o indicado na teoria, de que a inovação segue uma trajetória pré-estabelecida (path-dependence).

Para Redding (2002), cada inovação desenvolvida lança um conjunto de oportunidades para o desenvolvimento de inovações secundárias e essa trajetória histórica do desenvolvimento tecnológico influencia, inclusive, os incentivos que os agentes buscam ao tentarem desenvolver novas tecnologias.

A variável que representa os gastos públicos com $C \& T\left(C T_{t-1}\right)$ defasados temporalmente além de apresentar sinal negativo, não apresentou significância estatística. Esperava-se que os gastos com C\&T influenciassem positivamente a geração de inovação, entretanto esse resultado invalida esta hipótese nesta pesquisa.

Pode-se presumir que estes gastos sejam, sobretudo, em pesquisas acadêmicas que não chegam a gerar inovação. No trabalho de Co (2002), resultado similar foi encontrado e o autor conclui que o P\&D acadêmico não contribui para inovação americana. Tal resultado pode ser justificado também pelo próprio sistema de inovação brasileiro que, segundo Golçaves (2007), é centrado em segmentos tecnológicos de média e baixa intensidade e pouco baseado em gastos em $\mathrm{P} \& \mathrm{D}$ realizados pelas firmas. Assim, esse tipo de gasto não tem impacto na geração de inovação pelos estados.

A externalidade de diversificação, medidas pela variável $\left(D_{t}\right)$, apresentou o sinal negativo esperado, embora não seja estatisticamente significativa. Essa variável se comportou de maneira diversa entre os modelos estimados e em alguns, como é possível perceber na Tabela 6, se apresentou estatisticamente significativa.

As externalidades de especialização foram representadas pela variável $\left(S_{t}\right)$, que não apresentou o sinal esperado, mas é estatisticamente significativa. Isso mostra que, estudando o recorte estadual do país, externalidades de especialização industrial dificultam a geração de inovação. Logo, no período em análise, é possível rejeitar a hipótese do tipo MAR de que estruturas produtivas mais especializadas propiciariam a inovação regional.

Tanto o índice de especialização, quanto o índice de diversificação, mostram não impactar positivamente a geração de inovação nos estados brasileiros. Pelo fato de que, em alguns modelos estimados, o coeficiente da variável $\left(D_{t}\right)$ ter sido positivo, é presumível que haja a influência positiva da diversificação produtiva na geração de inovação e que a influência da especialização seja negativa neste período em análise.

A variável $\left(E_{t}\right)$ que mede a dotação de capital humano através do número de matrículas no ensino superior, apresentou o sinal positivo esperado e significância estatística. Quanto às variáveis defasadas espacialmente, o efeito do transbordamento desta última variável $\left(E_{t}\right)$ é positivo e estatisticamente significativo. Ou seja, o aumento de capital humano dos vizinhos influencia 
diretamente na geração de inovação dos estados.

O coeficiente da variável que mede o transbordamento da variável de inércia temporal da inovação $\left(W_{-} I_{t-1}\right)$ é positivo e estatisticamente significativo, revelando que as trajetórias préestabelecidas da inovação não ficam contidas apenas no território em que foram geradas, mas transbordam aos vizinhos.

A variável inovação defasada espacialmente $\left(W_{-} I_{t}\right)$ apresentou sinal positivo e significância estatística, o que demonstra que a atividade inovativa em vizinhos afeta positivamente a inovação nos estados brasileiros. A proximidade geográfica pode ser a influência espacial mais relevante nesse sentido, assim como o compartilhamento de infra-estrutura e concentração de mão-de-obra qualificada para a localização das firmas e instituições que atuam na geração de inovação no Brasil. Outras razões para que esse efeito transbordamento seja positivo são: o acesso da grandes mercados consumidores e aglomeração de indústrias em segmentos similares.

As outras variáveis defasadas espacialmente $\left(W_{-} C T_{t-1}, W_{-} S_{t}, W_{-} D_{t}\right)$ não apresentaram significância estatística e apresentaram sinal negativo em seus coeficientes. Isso mostra que, para este caso, os efeitos spillovers dessas variáveis não são relevantes e não impactam na geração de inovação dos estados brasileiros.

Para Gonçalves e Almeida (2009), o Brasil tem um sistema nacional de inovação que se caracteriza pela imaturidade, uma grande parte de patentes depositadas por pessoas físicas, um carácter ocasional de atividades inovadoras, um caráter adaptável e incremental das inovações, uma importante influência das empresas multinacionais, em comparação com empresas nacionais, uma parcela desproporcional das pequenas empresas em atividades de patenteamento e um baixo grau de interrelações entre os agentes constitutivos do sistema nacional de inovação, como as relações universidade-empresa.

Essas características podem ter influenciado os resultados dessa pesquisa, uma vez que os dados agregados por estado podem dificultar a pormenorização de situações diferentes do geral, como a ocorrência de "ilhas" tecnológicas espalhadas pelo Brasil, que possivelmente apresentariam resultados diferentes destes.

Através da AEDE, pode-se perceber que o padrão de associação espacial não se alterou significativamente durante o período em análise. A persistência desse padrão durante os onze anos observados comprova que o efeito de vizinhança foi determinante na manutenção de altos níveis de geração de inovação. Cabe observar que, embora existam meios legais de incentivo à inovação tecnológica para os estados das outras regiões do país, não houve movimento na distribuição espacial 
da inovação no Brasil.

Entre os resultados não esperados, a ausência de efeitos positivos vindos das externalidades de especialização e dos gastos públicos com C\&T configuram situações que podem, contudo, ser justificadas pelo arranjo da geração de inovação no Brasil.

Presume-se de parte dos resultados que o espaço exerce influência positiva e relevante para as inovações brasileiras. A inércia temporal indica que, uma vez estabelecidos mecanismos de geração de inovação em determinado estado ou região, a mudança espacial da mesma se torna difícil justamente porque a persistência temporal irá atuar na continuidade do processo inovativo naquele estado ou região. Esse pode ser um dos motivos pelos quais as políticas de incentivo à geração de inovação em outras localidades do país não tenham obtido sucesso durante os anos sob análise, persistindo a concentração de inovação nos estados do Sul e Sudeste.

Outra conclusão que os resultados permitem é a de que os gastos públicos, ao abrangerem majoritariamente investimentos em $\mathrm{P} \& \mathrm{D}$ acadêmicos, não promovem com entusiasmo a geração de inovação efetiva. Ainda que como inovação efetiva sejam considerados os controversos depósitos de patentes, que podem não captar todo o esforço que o país faz em prol da inovação. Em outras palavras, a difusão tecnológica através do espaço não consegue permitir que haja uma mudança espacial da inovação no Brasil.

Além do esforço em tentar difundir a atividade inovativa no país, existem incentivos para que as firmas sejam os principais atores na busca por patamares mais altos de geração de inovação. A Lei de Inovação Federal (Lei Federal n. ${ }^{\circ} 10.973$ de 02.12.2004) estabelece diversas formas de incentivo para que empresas privadas tornem-se inovadoras e estabeleçam bons indicadores de P\&D.

A pesquisa pode avançar nesse sentido, estabelecendo variáveis que reflitam os esforços próinovação e especificando modelos que satisfaçam a realidade distinta de um país como o Brasil. A literatura caminha para que o desenho do sistema de inovação brasileiro seja delimitado, e certamente, com o auxílio de dados mais abrangentes sobre pesquisa e inovação, as potencialidades e fragilidades serão apontadas e a sugestão de políticas públicas visando o aumento da inovação serão mais concisas e eficientes. 


\section{REFERÊNCIA}

Acs, Z. J., \& Audretsch, D. B. (1989). Patents as measure of innovative activity. Kyklos- International Review for Social Sciences, 42(2), 171-180.

Acs, Z. J., Audretsch, D. B., \& Feldman, P. (1994). R\&D spillovers and recipient firm size. The review of Economics and Statistics, 76(2), 336-340.

Albuquerque, E. M. (1999). National systems of innovation and Non-OECD countries: notes about a rudimentary and tentative "typology". Brazilian Journal of Political Economy, 19(4), 35-52.

Almeida, E. (2012). Econometria Espacial Aplicada. Campinas, SP: Editora Alínea.

Anselin, L. (1988). Spatial Econometrics: Methods and Models. Dordrecht: Studies in Operational Regional Science, Kluwer Academic, Boston.

Anselin, L. (1996). The Moran Scatterplot as an ESDA tool to assess local instability in spatial association. Spatial Analitycal perspectives in GIS. Taylor and Francis, London, 111-125.

Anselin, L. (1991). Interactive techniques and exploratory spatial data analysis. Geographical Information Systems: principles, techniques, management and applications, 1, 251-264.

Anselin, L., \& Bera, A. K. (1998). Spatial dependence in linear regression models with an introduction to Spatial Econometrics. In A. Ullah and D. Giles (Eds), Handbook of Applied Economic Statistics, Berlin.

Arrow, K. (1989). The economic implications of learning by doing. Economic Studies, 29, 155-173.

Arthur, B. (1989). Competing technologies, increasing returns and lock in by historical events. The Economic Journal, 99(394), 116-131.

Audretsch, D. B., Feldman, M. P. (1996). R\&D Spillovers and the geography of innovation and production. American Economic Review, 86(3), 630-640.

Audretsch, D. B. (1998). Agglomeration and the location of innovative activity. Oxford. Review of Economic Policy, 14(2).

Audretsch, D. B., Aldridge, T. T. (2010). Knowledge spillovers, entrepreneurship and regional development. Handbook of regional growth and development theories, 201.

Badinger, H., \& Muller, W. G., \& Tondl, G. (2004). Regional convergence in the European Union (1985-1999): a spatial dynamic panel analysis. Regional Studies, 58, 241-253.

Baller, R. D. et al. (2001). Structural covariates of us county homicide rates: incorporating spatial effects*. Criminology, 39(3).

Baumont, C., Ertur, C., \& Le Gallo, J. (2002). Spatial convergence clubs and the European regional growth process, 1980-1995. In European regional growth. Springer Berlin Heidelberg.

Revista de Administração e Inovação, São Paulo, v. 12, n.3 p. 109-134, jul./set. 2015. 
Bergek, A., \& Norrman, C. (2008). Incubator best practice: A framework. Technovation, 28(1), 20-28.

Bilbao-Osorio, B., \& Rodríguez-Pose, A. (2004). From R\&D to Innovation and Economic Growth in the EU. University of Kentucky. Growth and Change, 35(4).

BRASIL. Lei de Inovação Tecnológica (Lei n. ${ }^{\circ}$ 10.973/2004). Brasília, DF: Congresso Nacional. Atos do Poder Legislativo, DOU de 03.12.2004.

BRASIL. Lei do Bem (Lei no. 11.196/2005). Brasília, DF: Congresso Nacional. Atos do Poder Legislativo, DOU de 21.11.2005.

Cabre-Borrás, B., \& Serrano-Domingo, G. (2007) Innovation and R\&D spillover effects in Spanish regions: A spatial approach. Research Policy, 36(9), 1357-1371.

Cameron, A. C., \& Trivedi, P. K. (2005). Microeconomics: Methods and Applications. Cambridge University Press, Cambridge.

Cardoso, L. C. B., Bittencourt, M. V. L., \& Porsse, A. A. Demanda por Combustíveis Leves no Brasil: uma abordagem utilizando painéis espaciais dinâmicos. XLI Encontro Nacional de Economia 2012

Castaldi, C., Dosi, G., \& Paraskevopoulou, E. (2011). Path dependence in technologies and organizations: a concise guide. LEM Working Paper Series.

Cohen, W., \& Levinthal, D. (1986). Spatial econometrics and political science. In annual Meeting of the Southern Political Science Association, Atlanta: Department of Political Science, University of South Carolina, Columbia.

De Negri, J. A., Salermo, M. S., \& Castro, A. B. (2005). Inovações, padrões tecnológicos e desempenho das firmas industriais brasileiras. In: De Negri, J. A., Salermo, M. S. (Orgs.), Inovações, padrões tecnológicos e desempenho das firmas industriais brasileiras. Brasília: IPEA, $5-46$.

Dosi, G. (1988). Sources, procedures, and microeconomic effects of innovation. Journal of Economic Literature, 26, 1120-1171.

Diniz, C. C., \& Gonçalves, E. (2001). Economia do conhecimento e desenvolvimento regional no Brasil. In I Encontro de Estudos Regionais e Urbanos. São Paulo.

Elhorst, J. P. (2003). Specification and estimation of spatial panel data models. International Regional Science Review. 3(26), 244-268.

Feldman, M. P. (1994). Knowledge complementarity and innovation. Small Business Economics, 6(5), 363-372.

Feldman, M. P., \& Audretsch, D. B. (1999). Innovation in cities: Science-based diversity, specialization and localized competition. European economic review, 43(2), 409-429. 
Fischer, M. M. (2001). Innovation, knowledge creation and systems of innovation. The Annals of Regional Science, 35(2), 199-216.

Glaeser, E. L., Kallal, H. D., Scheinkman, J. A., \& Shleifer, A. (1992). Growth in cities. Journal of Political Economy, 100(6).

Gonçalves, E. (2007a) Firma e território: três ensaios sobre inovação em ambientes periféricos (Tese de doutorado). Universidade Federal de Minas Gerais, Belo Horizonte.

Gonçalves, E. (2007b). O padrão espacial da atividade inovadora brasileira: uma análise exploratória. Estudos Econômicos (São Paulo), 37(2), 405-433.

Gonçalves, E., \& Almeida, E. S. (2008). Innovation and spatial knowledge spillovers: evidence from Brazilian patent data. In 8th RSAI WORLD CONGRESS, São Paulo.

Griliches, Z. (1979). Issues in assessing the contribution of research and development to productivity growth. Bell Journal of Economics, 10, 92-116.

Gujarati, D. N. (2006). Econometria básica. 4. ed. Rio de Janeiro: Elsevier.

Jacobs, J. (1969). The economy of cities. Nova York: Random House.

Jaffe, A. B., Henderson, R. M., \& Trajtenberg, M. (1993). Geographic localization of knowledge spillovers as evidenced by patent citation. Quarterly Journal of Economics, 63(3), 577-598.

Koo, J. (2005). Agglomeration and spillovers in a simultaneous framework. The Annals of Regional Science, 39.

Lemos, M. B., Moro, S., Domingues, E. P., \& Ruiz, R. M. A. (2005). Espaços preferenciais e aglomerações industriais. In Negri, J. A. \& Salermo, M. (ed.). Inovação, Padrões Tecnológicos e Desempenho das Firmas Industriais Brasileiras. Brasília: IPEA, 365-424.

Lesage, J., \& Pace, R. K. (2014). The biggest myth in spatial econometrics. Econometrics, 2, 217-249.

Luna, F., Baessa, A. (2007). Impacto das marcas e das patentes no desempenho econômico das firmas. In Anais do XXXV Encontro Nacional de Economia. ANPEC-Associação Nacional dos Centros de Pós-graduação em Economia.

Organisation for Economic Co-operation and Development. (2002). Manual Frascati. Proposed Standard Practice for Surveys on Research and Experimental Development.

Marshall, A. (1982). Princípios de Economia. São Paulo: Abril Cultural.

MCTI. (2013). Indicadores Estaduais de Ciência \& Tecnologia. Brasília. Retirado de http://www.mct.gov.br/riecet_indicadores_estaduais. 
Montenegro, R. L., Gonçalves, E., \& Almeida, E. (2011). Dinâmica espacial e temporal da inovação no estado de São Paulo: uma análise das externalidades de diversificação e especialização. Estudos Econômicos, 41(4).

Nijkamp, P., Siedschlag, I., \& Smith, D. (2011). Economic Growth, Innovation and Competitiveness in a Knowledge-Based World Economy: Introduction. In Innovation, Growth and Competitiveness. Springer Berlin Heidelberg, 1-11.

Paci, R., \& Usal, S. (2000). The role of specialization and diversity externalities in the agglomeration of innovative activities. Rivista Italiana degli Economisti, 2(2), 237-268.

Pacheco, C. A. (2003). As reformas da política nacional de ciência, tecnologia e inovação no Brasil (1999-2002). Documento preparado para la Comisión Económica para América Latina y el Caribe (CEPAL), Campinas, Brasil.

Pavitt, K. (1985). Sectoral patterns of technical change: towards a taxonomy and a theory. Research Policy, 13(6), 343-373.

Pavitt, K., \& Patell, P. (1991). Large firms in the production of the world's technology: an important case of non-globalisation. Journal of International Business Studies, 22, 1-21.

Peters, L., Rice, M., \& Sundararajan, M. (2004). The role of incubators in the entrepreneurial process. The Journal of Technology Transfer, 29(1), 83-91.

Penrose, E. (1973). International patenting and the less-developed countries. The Economic Journal, 83(331), 768-786.

Phillips, R. G. (2002). Technology business incubators: how effective as technology transfer mechanisms? Technology in Society, 24(3), 299-316.

Porter, M. (1990). The competitive advantage of nations. Londres: Macmillan.

Redding, S. (2002). Path Dependence, Endogenous Innovation, and Growth. International Economic Review, 43(4).

Romer, P. (1990). Endogenous technological change. Journal of Political Economy, 98 (2), 71-102.

Salerno, M. S., \& Kubota, L. C. (2008). Estado e inovação. Políticas de incentivo à inovação tecnológica. Brasília: Ipea.

Schumpeter, J. A. (1961). Teoria do desenvolvimento econômico. Fundo de Cultura.

Sen, M., \& Bera, A. K. (2011). Specification Testing for Panel Spatial Models. Working Paper of University of Illinois at Urbana Champaing.

Vargas, M. A. (2002). Proximidade territorial, aprendizado e inovação: um estudo sobre a dimensão local dos processos de capacitação inovativa em arranjos e sistemas produtivos no Brasil (Tese de Doutorado). Instituto de Economia, Universidade Federal do Rio de Janeiro, Rio de Janeiro, 2002. 
Vieira, R. S. (2009). Crescimento econômico no estado de São Paulo: uma análise espacial [online]. São Paulo: Cultura Acadêmica.

Wooldridge, J. M. Econometric analysis of cross section and panel data. The MIT Press: Massachusets.

\title{
DIVERSIFICATION AND EXPERTISE PRODUCTION IN TECHNOLOGICAL INNOVATION GENERATION: AN APPLICATION FOR BRAZILIAN STATES
}

\begin{abstract}
The main objective of this research is to investigate how sensitive is the generation of innovation to the influences of the Brazilian states of externalities of industrial diversification and industrial specialization in the period 2001-2011. Furthermore, studying the behavior of innovation through the territory allows the inference of how public policies to encourage science and technology have acted in Brazil. Other determinants of innovation are also considered as the investment capacity of the states in science and technology, education level and lag of innovation. As a generation of innovation, deposits of patents per capita are used in order to measure the technological capacity of each state. The database used in this work consists of a combination of data from the Brazilian Institute of Geography and Statistics (IBGE), data of patent applications at the National Institute of Industrial Property (INPI), data on spending on science and technology from the Ministry of Science, Technology and Innovation (MCTI) and data on human capital of the Ministry of Education (MEC). The methodology addresses the Exploratory Spatial Data Analysis (ESDA) and spatial regression models with panel data. These procedures permitted to follow the trajectory of innovation through the territory in the period and the main legal framework of the Brazilian innovation were studied in order to establish if these efforts contributed to the strengthening of regional innovation policy.
\end{abstract}

Keywords: Innovation; Patents; Brazilian states; models in panel data with spatial dependence.

Data do recebimento do artigo: 05/07/2014

Data do aceite de publicação: 18/06/2015

Revista de Administração e Inovação, São Paulo, v. 12, n.3 p. 109-134, jul./set. 2015. 